\title{
Multi-microscopy nanoscale characterization of the doping profile in a hybrid
}

\section{$\mathrm{Mg} / \mathrm{Ge}$-doped tunnel junction.}

E. Di Russo ${ }^{1 *}$, A. Mavel ${ }^{1}$, V. Fan Arcara ${ }^{1,2}$, B. Damilano ${ }^{2}$, I. Dimkou ${ }^{1}$, S. Vézian ${ }^{2}$, A. Grenier ${ }^{1}$, M. Veillerot ${ }^{1}$, N. Rochat $^{1}$, G. Feuillet ${ }^{1}$, B. Bonef ${ }^{4}$, L. Rigutti ${ }^{5}$, J-Y. Duboz ${ }^{2}$, E. Monroy ${ }^{3}$, D. Cooper ${ }^{1}$

${ }^{1}$ Univ. Grenoble Alpes, CEA, LETI, F-38000 Grenoble.

${ }^{2}$ CNRS, Université Côte d'Azur, CRHEA, Rue Bernard Grégory, 06560 Valbonne, France.

${ }^{3}$ Univ. Grenoble-Alpes, CEA, IRIG-PHELIQS, 17 av. des Martyrs, 38000 Grenoble, France.

${ }^{4}$ Materials Department, University of California Santa Barbara, CA, 93106, USA.

${ }^{5}$ UNIROUEN, INSA Rouen, CNRS, Groupe de Physique des Matériaux, Normandie Université, 76000 Rouen, France.

${ }^{*}$ Corresponding author: enrico.dirusso@cea.fr

\begin{abstract}
A multi-microscopy investigation of a GaN tunnel junction (TJ) grown on an InGaN-based light emitting diode (LED) has been performed. The TJ consists of a heavily Ge-doped n-type GaN layer grown by ammonia-based molecular-beam epitaxy on a heavily Mg-doped p-type GaN thin layer, grown by metalorganic vapor phase epitaxy. A correlation of atom probe tomography, electron holography and secondary ion mass spectrometry has been performed in order to investigate the $\mathrm{nm}$-scale distribution of both $\mathrm{Mg}$ and $\mathrm{Ge}$ at the TJ. Experimental results reveal that $\mathrm{Mg}$ segregates at the $\mathrm{TJ}$ interface, and diffuses into the Ge-doped layer. As a result, the dopant concentration and distribution differ significantly from the nominal values. Despite this, electron holography reveals a TJ depletion width of $\sim 7 \mathrm{~nm}$, in agreement with band diagram simulations using the experimentally determined dopant distribution.
\end{abstract}

Keywords: atom probe tomography, electron holography, tunnel junction, Mg-doping, Ge-doping, LED. 


\section{Introduction}

Light emitting diodes (LEDs) based on $\ln _{x} \mathrm{Ga}_{1-\mathrm{x}} \mathrm{N} / \mathrm{GaN}$ multi-quantum wells (MQWs) are widely used as solidstate light sources [1]. The efficiency of these devices has been limited during many years by $p$-doping issues, due to the large activation energy of the $\mathrm{Mg}(\sim 185 \mathrm{meV})$, the shallowest acceptor known for nitrides [2]. These problems have been solved to a satisfactory level as best blue LEDs currently have outstanding efficiencies. However, such issues remain when dealing with LEDs emitting at shorter wavelengths. For these UV LEDs, and for visible LEDs in special structures where several junctions are stacked on top of each other, tunnel junctions (TJ) have been proposed and were demonstrated to provide a good solution to the problem [3-7]. The TJ essentially consists of a degenerately doped $p$-n junction placed on top of the p-layer of the emitting junction, which allows effective band-to-band tunneling under reverse bias (corresponding to forward bias of the light emitting junction). The adoption of the TJ architecture mitigates the limited current spreading and reduces the contact resistance (the contact resistance of the metal $/ \mathrm{n}^{+}-\mathrm{GaN}$ scheme is significantly lower than that of metal/p-GaN), without degradation of the light extraction efficiency [8-9]. Another potential advantage is the possibility to interconnect multiple active regions in cascade LEDs, in order to circumvent efficiency droop [10-11]. Finally, recent developments of growth techniques allow for the replacement of $\mathrm{Si}$ with $\mathrm{Ge}$ as the n-type dopant in $\mathrm{GaN}$, achieving n-type doping levels higher than $1 \times 10^{20} \mathrm{~cm}^{-3}$ with smoother surfaces and lower film stress than for the case of Si-doped GaN [12-15].

The challenges to implement a TJ architecture in wide bandgap materials (i.e. GaN) are strongly linked to the need to reach a sufficiently high interband tunneling probability across the depletion region. Such probability decreases exponentially with increasing TJ depletion width $w_{d}$ [16] which is given at zero bias by the following relation [16-18]

$$
w_{d} \approx \sqrt{\frac{2 \varepsilon V_{b i}\left(N_{A}+N_{D}\right)}{e N_{A} N_{D}}},
$$

where $\varepsilon$ is the material permittivity $\left(9.8 \varepsilon_{0}\right.$ for $\mathrm{GaN}$, with $\varepsilon_{0}$ the vacuum permittivity [19]), $V_{b i}$ is the built-in potential $(\approx 3.25 \mathrm{eV}$ for $\mathrm{GaN}), N_{A}$ and $N_{D}$ are the substitutional doping concentrations of acceptors and donors, respectively, and $e$ is the electron charge. As an example, with $N_{A}=1 \times 10^{20} \mathrm{~cm}^{-3}$ and $N_{D}=5 \times 10^{20}$ $\mathrm{cm}^{-3}$ (nominal doping of the diode studied in this article), $w_{d}$ is equal to $7 \mathrm{~nm}$.

In GaN-based devices, the high built-in potential $\left(V_{b i}\right)$ and the solubility limit of the dopants make it difficult to attain high tunnel probabilities. Another specific issue arises from the passivation of $\mathrm{Mg}$ by hydrogen during the growth by metal organic chemical vapor deposition (MOCVD). Even if $\mathrm{Mg}$ can be activated by annealing after the p-layer growth [20-21], it is at least partially re-passivated during the subsequent growth of the $n$-layer on top of the p-layer to fabricate the TJ. A new approach consists of growing the $\mathrm{n}^{+}$side of the TJ by ammonia-based molecular-beam epitaxy ( $\left.\mathrm{NH}_{3}-\mathrm{MBE}\right)$ directly on an LED device synthesized 
by MOCVD $[7,22]$. This two-step growth allows post-growth annealing after the MOCVD growth. Then, during the overgrowth of $n$-doped GaN, the relatively low partial pressure of $\mathrm{H}_{2}$ in the $\mathrm{NH}_{3}-\mathrm{MBE}$ system prevents the re-passivation of the $\mathrm{p}-\mathrm{GaN}$ layer.

The aim of the present study is to correlate the chemical, structural and electrical properties of a $\mathrm{Mg} / \mathrm{Ge}$ doped TJ grown on top of a $\ln _{x} G_{1-x} N / G a N-b a s e d ~ L E D$. It has already been demonstrated that this architecture leads to an enhancement of the LED emission [22]. However, the in-depth understanding of the tunneling mechanisms requires an assessment of the distribution of dopants at the $p / n$ interface, i.e. the MOCVD/MBE interface. A comparative multi-microscopy approach based on atomic force microscopy (AFM), off-axis electron holography, secondary ion mass spectrometry (SIMS) and laser-assisted atom probe tomography (La-APT) was adopted to obtain information about the structural properties, dopant distribution and activation. The interest of the present work is two-fold. First, it provides operational guidelines for an accurate analysis of dopant distribution at the $\mathrm{nm}$ scale, showing possible strengths and limitations of the different techniques. Then, the experimental results obtained represent a significant gain in the understanding of diffusion and segregation phenomena of dopants, with impact on the electrical properties of GaN-based TJs.

\section{Materials and methods}

A schematic of the LED stack studied here is shown in fig. 1(a). The LED structure up to the $\mathrm{p}^{++}$-GaN layer was grown along the [0001] direction on a 2" sapphire substrate in an MOCVD reactor. The precursors of $\mathrm{Ga}$, In, Al, Mg, Si and N were trimethylgallium (TMGa) and triethylgallium (TEGa), trimethylindium (TMIn), trimethylaluminium (TMAl), bis(cyclopentadienyl)magnesium $\left(\mathrm{Cp}_{2} \mathrm{Mg}\right)$ and ammonia $\left(\mathrm{NH}_{3}\right)$, respectively. The nominal stacking sequence starts with a $1 \mu \mathrm{m}$ non-intentionally doped (nid) GaN layer, followed by 2 $\mu \mathrm{m}$ Si-doped GaN ([Si] $\left.=6 \times 10^{18} \mathrm{~cm}^{-3}\right)$. The active region consists of $5 \ln _{x} G_{1-x} N / G a N(6 \mathrm{~nm} / 12 \mathrm{~nm})$ quantum wells. Then, a 20-nm-thick $\mathrm{Al}_{\mathrm{y}} \mathrm{Ga}_{1-\mathrm{y}} \mathrm{N}$ electron blocking layer (EBL) was grown, followed by 110-nmthick Mg-doped GaN layers (100 nm with $[\mathrm{Mg}]=3 \times 10^{19} \mathrm{~cm}^{-3}$ and $10 \mathrm{~nm}$ with $\left.[\mathrm{Mg}]=1 \times 10^{20} \mathrm{~cm}^{-3}\right)$. The MOCVD growth of the Mg-doped GaN layers was performed at $975^{\circ} \mathrm{C}$. The LED was then annealed for 20 min at $700{ }^{\circ} \mathrm{C}$ under $\mathrm{N}_{2}$ atmosphere to activate the $\mathrm{Mg}$ acceptors. The wafer was transferred to an MBE Riber reactor equipped with $\mathrm{Ga}$ and $\mathrm{Ge}$ solid sources. The $\mathrm{NH}_{3}-\mathrm{MBE}$ growth of the Ge-doped GaN layers (20 $\mathrm{nm}$ with $[\mathrm{Ge}]=5 \times 10^{20} \mathrm{~cm}^{-3}, 200 \mathrm{~nm}$ with $[\mathrm{Ge}]=1 \times 10^{19} \mathrm{~cm}^{-3}$ ) was performed at $790{ }^{\circ} \mathrm{C}$. The opto-electrical properties of the LED are described elsewhere $[22,23]$. 
Theoretical calculations of the band profile and electronic levels were performed using the Nextnano software [24] with the material parameters reported in ref. [25]. This software solves the Schrödinger and Poisson equations in a self-consisted manner, using the $\mathrm{k} \cdot \mathrm{p}$ model. Calculations were performed in one dimension and with zero bias, assuming that the structure grows pseudomorphically on GaN. Varshni equation is used to calculate the semiconductor bandgap, considering room temperature conditions. Spontaneous and piezoelectric polarization, as well as the band gap deformation potentials, are taken into account. The simulated band diagram corresponding to the nominal LED structure is shown in fig. 1(b). Starting from the bottom of the structure, the GaN:Si and MQW regions have relatively flat bands, with the potential at the junction dropping mainly in the AIGaN EBL. This behavior is characteristic of III-nitride band profiles and stems from the large difference in polarization between AIGaN and GaN. Also related to polarization, the MQW present a saw-tooth profile that is due to the internal electric fields generated mostly by piezoelectric polarization in the InGaN wells, with a contribution of the difference in spontaneous polarization between GaN and InGaN. The bands remain also flat in the GaN:Mg area, since the de high density of incorporated $\mathrm{Mg}$ blocks the extension of the depletion region above the EBL. At the interface between GaN:Mg and GaN:Ge, the high doping levels result in the conduction and valence band getting close to each other, which should allow carrier transport by tunnel between bands. Calculations of the quantum-confined electron and hole levels in the MQWs predict an optical transition at $431 \mathrm{~nm}$, in good agreement with experimental observations (peak emission at $436 \mathrm{~nm}$ ) [23].

(a)

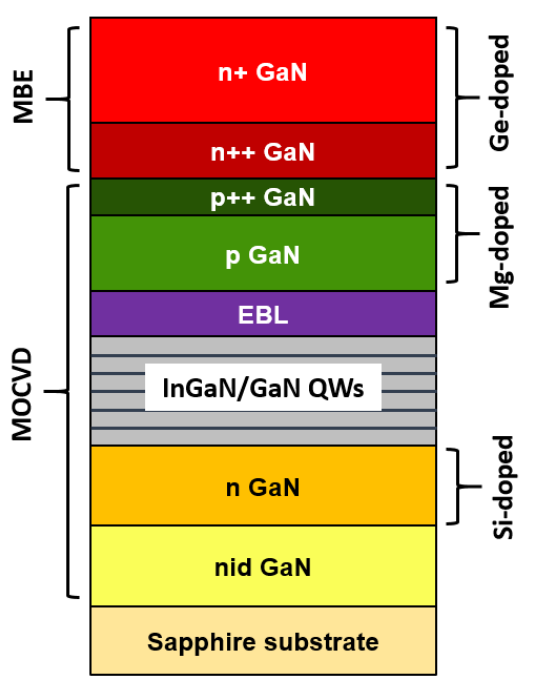

(b)

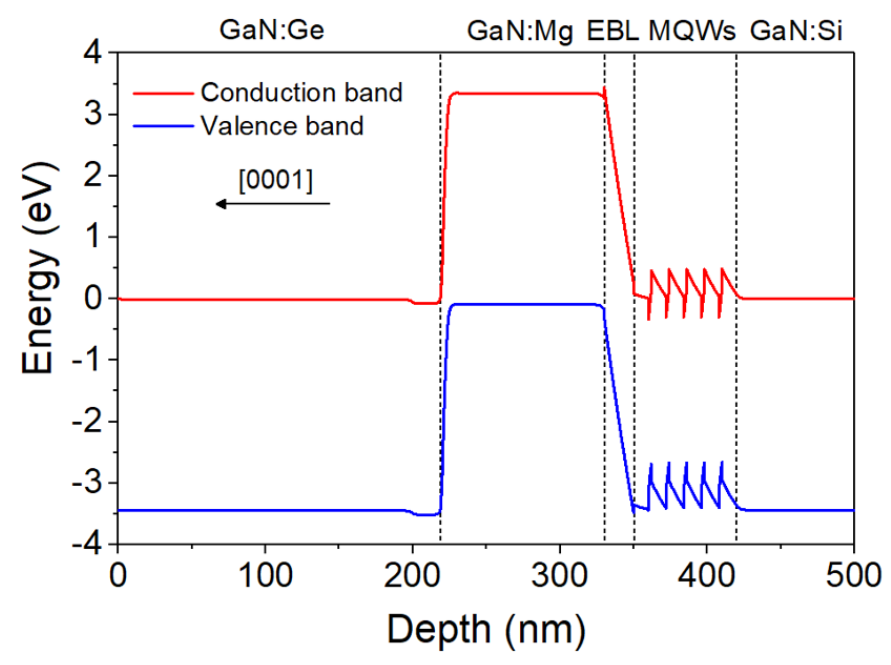

Fig. 1: (a) Schematic of the blue $I n_{y} G a_{1-y} N / G a N$-based LED. (b) Band diagram simulation of the structure in fig. 1(a). 
To assess the sample morphology, AFM measurements were performed using a Dimension Icon AFM system operated in a tapping mode using Bruker TESPA-V2 tips.

A transmission electron microscopy (TEM) lamella specimen and needle-shaped tips required for La-APT were prepared using a FEI Helios NanoLab 450S scanning electron microscope/focused ion beam (SEM/FIB). The specimen preparation method consisted in a standard lift-out procedure followed by tip sharpening with $16 \mathrm{kV} \mathrm{Ga}$ ions and a final cleanup process at $2 \mathrm{kV}$, in order to reduce the thickness of the damaged volume [26-28].

HAADF-STEM observations were carried out along the [11 $\overline{2} 0]$ direction of the lamella specimen using an aberration corrected FEI Titan Themis operated at $200 \mathrm{kV}$. Instead, electron holography was performed along the [0120] direction using FEI Titan Ultimate operated at $200 \mathrm{kV}$. To improve the phase resolution stacks of electron holograms were recorded and then reconstructed using the Holoview software [29].

SIMS investigations were performed using a IMS magnetic selector instrument from CAMECA [30]. Due to the different chemical nature of $\mathrm{Mg}$ and Ge atoms, two measurements were carried out with optimized relative ion yields. Both $\mathrm{Mg}$ and Ge concentration profiles were then calculated.

Laser-assisted atom probe tomography (La-APT) was performed using a CAMECA FlexTAP system, operated at $40 \mathrm{~K}$ with a UV femtosecond laser $\left(344 \mathrm{~nm}\right.$, repetition rate $=50 \mathrm{kHz}$, pulse peak energy $\left.=2.5 \times 10^{-4} \mathrm{~J} \cdot \mathrm{cm}^{-2}\right)$. The field of view was set to $15^{\circ}$ and detection rate to $0.0035-0.0050$ event/pulse. Data were processed using the Tap3D software from CAMECA and homemade MATLAB codes. The 3D reconstructions were performed using a cone-angle algorithm [31].

\section{Results and discussion}

\section{(a) Scanning Transmission Electron Microscopy}

A high-angle annular dark-field scanning transmission electron microscopy (HAADF-STEM) image of the lamella specimen is shown in fig. 2(a). This imaging technique provides a signal intensity approximately proportional to the square of the average atomic number $Z^{2}$. Therefore five bright layers correspond to the $\ln _{x} G_{1-x} N$ quantum wells, while the darker layer is the $\mathrm{Al}_{y} \mathrm{Ga}_{1-y} \mathrm{~N}$ EBL. Energy dispersion $x$-ray spectroscopy (EDS) was then performed to obtain information about the Ge distribution. An EDS profile associated to the Ge K-line signal intensity extracted from the region indicated in fig. 2(a) along the [0001] growth direction is shown in fig. 2 (b). In such a profile, the Ge signal in the $\mathrm{n}^{+}$-layer remains below the detection threshold, but 
we clearly resolve the $\sim 20$-nm-thick heavily Ge-doped region, corresponding to the $\mathrm{n}^{++}$GaN layer that is placed $100 \mathrm{~nm}$ above the EBL (see fig. 1(a)). Here the Ge concentration has not been quantified due to the low signal-to-noise ratio. The EDS concentration profile of $\mathrm{Mg}$ cannot be detected here as the $\mathrm{Mg} \mathrm{K}$-line (1.25 keV) is very close to the Ga L-line (1.19 keV) [32].

(a)

\section{HAADF-STEM}

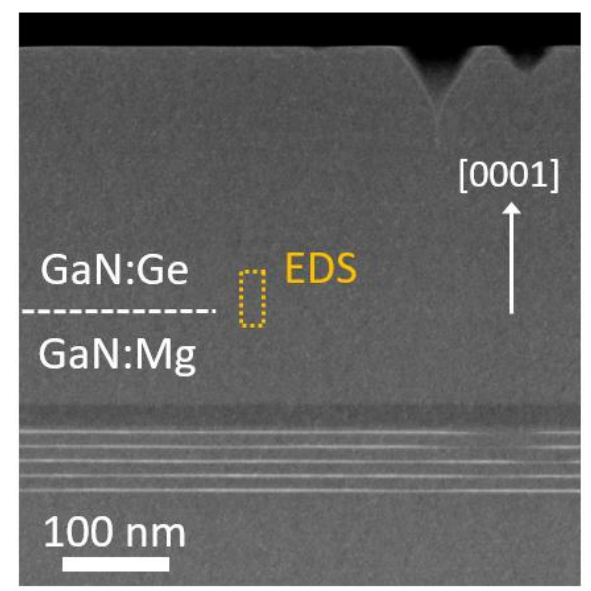

(b)

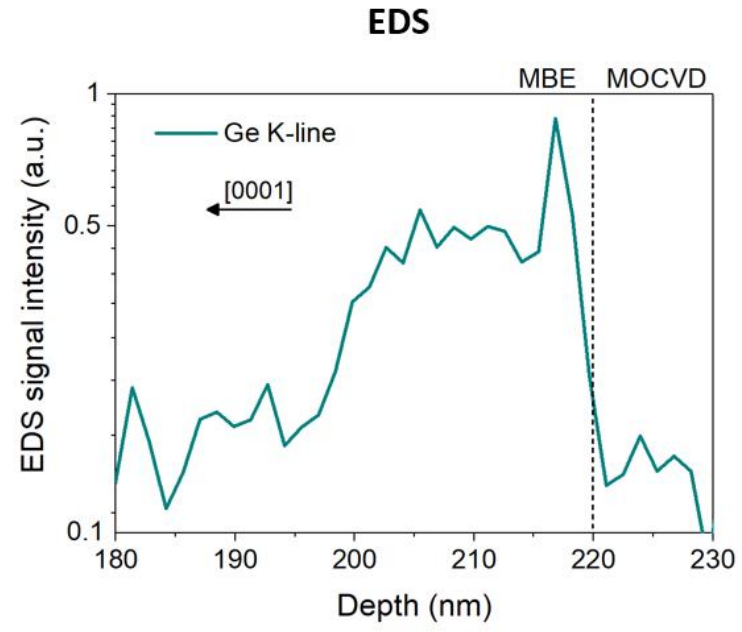

Fig. 2: (a) HAADF-STEM micrograph of the lamella specimen. Only the InGaN QWs (bright contrast) and the AIGaN EBL (dark contrast) can be distinguished from the GaN background. (b) EDS intensity profile of the Ge K-line that allows locating the MOCVD/MBE interface.

\section{(b) Atomic Force Microscopy}

The HAADF-STEM cross section image shows V-shaped pits (V-pits) at the surface, with a depth that can reach $\sim 50 \mathrm{~nm}$. This kind of pits, which are associated with threading dislocations as shown in fig. 2(a), are commonly observed in highly Si-doped GaN layers [33,34]. Replacing Si dopants with Ge results in a reduction of the local strain in the lattice, which generally reduces this kind of defects [35]. However, V-pits decorating dislocations are still observed in heavily Ge-doped GaN layers grown by MOCVD [35] or by NH3MBE [36]. In the sample under study, the depth of the V-pits observed by HAADF-STEM is smaller than the thickness of the Ge-doped GaN layer. Therefore, they should not have any effect on the transport properties of the tunnel junction. However, the presence of V-pits can cause artifacts in chemical measurements based on top-down profiling (e.g. SIMS). Therefore, we have studied the surface morphology at a larger scale using AFM. A $2 \times 2 \mu \mathrm{m}^{2}$ scan of the sample surface is displayed in fig. 3(a). It confirms that V-pits appear randomly distributed with a pit density $\approx 4 \times 10^{9} \mathrm{~cm}^{-2}$. Their typical hexagonal morphology can be clearly resolved in the AFM zoomed image. The V-pits size distribution is shown in fig. 3(b). The average V-pit width is about $70 \mathrm{~nm}$. It should be noted that this width measurement is reliable, 
but the average depth on them $(\sim 10 \mathrm{~nm})$ can be strongly underestimated here due to the geometry of the AFM tip. Therefore, the cross-section TEM investigation provides a more reliable indication of the V-pit depth. The presence of these pits strongly affects the root mean square surface roughness, which was found equal to $(2.5 \pm 0.7) \mathrm{nm}$ for the $2 \times 2 \mu \mathrm{m}^{2}$ AFM map. This value increases to $(3.9 \pm 0.7) \mathrm{nm}$ for a $20 \times$ $20 \mu \mathrm{m}^{2}$ scan. As a term of comparison, AFM investigations performed before the MBE growth reveal that the $\mathrm{p}^{++}$layer presented a very smooth surface with a roughness of $\sim 0.2 \mathrm{~nm}$.

(a)

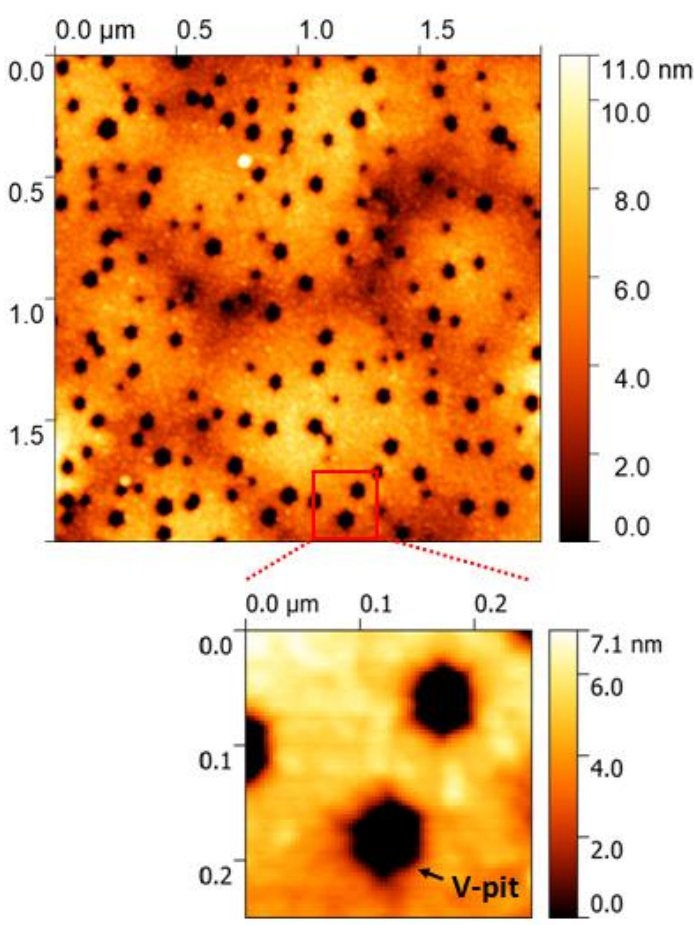

(b)

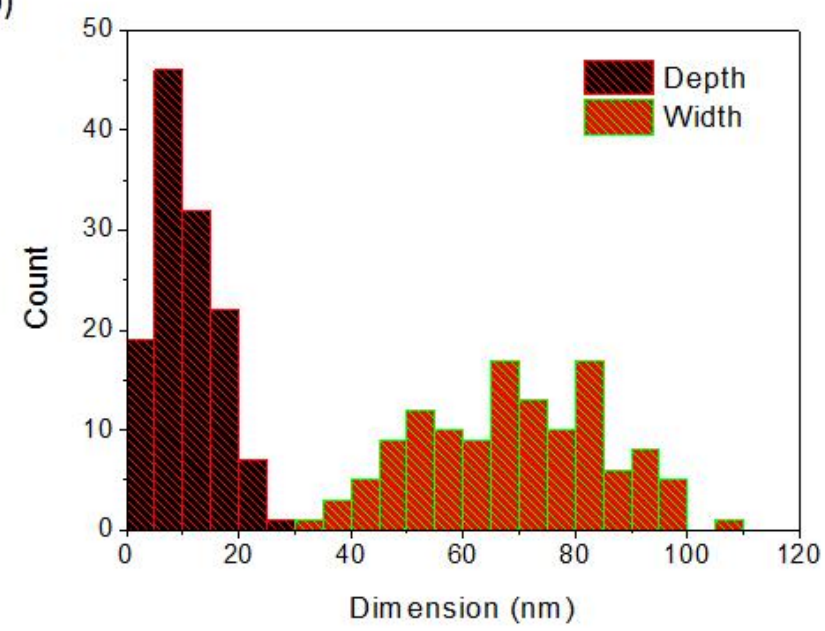

Fig. 3: (a) AFM map of the sample surface (tapping mode). The zoomed image corresponds to the $250 \times 250$ $\mathrm{nm}^{2}$ red box in the larger view. The color scale highlights the details of sample surface but does not allow measuring the interior of the V-pits. (b) Depth and width distribution of the V-pits extracted from fig. 3(a).

\section{(c) Secondary Ion Mass Spectrometry}

Precise quantification of the dopant concentrations was obtained by SIMS. The Mg- and Ge-doping profiles at the TJ are shown in fig. 4. The Ge concentration in the $n^{+}$GaN layer is equal to the nominal value $(1 \times$ $10^{19} \mathrm{~cm}^{-3}$ ). On the contrary, the measured Ge concentration the $\mathrm{n}^{++}$layer reaches only $2 \times 10^{20} \mathrm{~cm}^{-3}$, slightly lower than the targeted value. Then, in the first $30 \mathrm{~nm}$ of the $\mathrm{p}^{++}$layer, the Ge concentration decreases gradually to the noise level (few $10^{16} \mathrm{~cm}^{-3}$ ). This tail is most probably an artifact originated both by the surface roughness and/or by mixing effects during SIMS measurements, as a diffusion of Ge into the $p$ - 
doped region is thought to be unlikely in such a structure [37]. If we turn our attention to the distribution of $\mathrm{Mg}$, the measured concentration is $\sim 2 \times 10^{19} \mathrm{~cm}^{-3}$ in the $\mathrm{p}$ layer, increases up to $\sim 2 \times 10^{20} \mathrm{~cm}^{-3}$ in the $\mathrm{p}^{++}$ layer. Both concentrations are slightly higher than the targeted values. Finally, a tail of $\mathrm{Mg}$ penetrates into the $\mathrm{n}^{+} \mathrm{GaN}$ layer, decaying by one order of magnitude every $20 \mathrm{~nm}$ (i.e. the penetration of $\mathrm{Mg}$ into $\mathrm{n}^{+}-\mathrm{GaN}$ is too large to be an artifact). Out-diffusion of $\mathrm{Mg}$ can be promoted by the high substrate temperature (790 ${ }^{\circ} \mathrm{C}$ ) during the MBE growth. Another likely explanation is Mg segregation during the MOCVD growth, and a gradual incorporation of the $\mathrm{Mg}$ excess in the topmost area during the MBE growth. It is important to notice that SIMS measurements do not reveal any accumulation of oxygen or carbon at the $p / n$ interface (profiles not shown here), despite the change of growth reactor, which implied the exposure to air. All along the sample, the levels of oxygen and carbon remain below the detection limit of the setup $\left([\mathrm{O}]_{\lim }=5 \times 10^{19} \mathrm{~cm}^{-3} ;[C]_{\lim }=3 \times 10^{16} \mathrm{~cm}^{-3}\right)$.

It must be noted that SIMS is highly sensitive to the dopant concentration but can be limited in terms of depth resolution due to the roughening of GaN material specimen under ion irradiation. Broadening, distortions and an eventual shift of the concentration profiles are likely artifacts, which are enhanced in samples with as-grown rough surfaces. Here, the presence of V-pits inevitably affects the SIMS depth resolution, making it difficult to get accurate profiles for the Ge and Mg concentrations across the TJ interface.

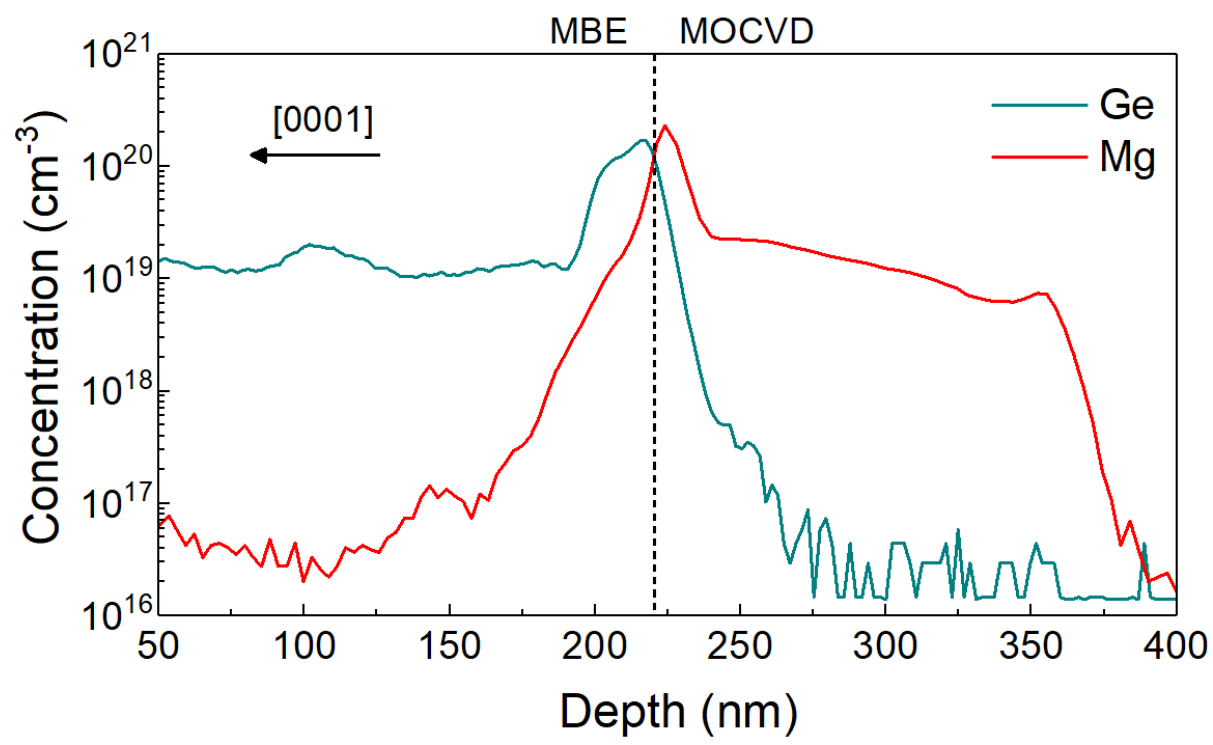

Fig. 4: Mg-and Ge-doping profiles measured by SIMS. The MOCVD/MBE interface is expected to be situated between the two main concentration peaks, at a $220 \mathrm{~nm}$ depth.

\section{(d) Laser-assisted Atom Probe Tomography}


The spatial distribution of dopants in correspondence of the TJ has been revealed by La-APT. A 3D reconstruction of the volume around the $\mathrm{TJ}$ is shown in fig. 5(a) where $\mathrm{Mg}^{2+}$ ions are represented in red, while $\mathrm{Ge}^{2+}$ ions are depicted in blue. The overall volume dimension represented here is $20 \times 30 \times 40 \mathrm{~nm}^{3}$, corresponding to $5.3 \times 10^{5}$ detected ions. For this $3 \mathrm{D}$ reconstruction, the initial tip curvature radius was set to $65 \mathrm{~nm}$ and the cone angle to $8^{\circ}$. These parameters correspond to the tip morphology measured by TEM prior to APT analysis. They allow the 3D reconstruction of the entire LED structure, showing flat interfaces and providing the nominal thicknesses of each layer.

Two distinct segments of the mass spectrum associated to the reconstructed volume are shown in fig. $5(b, c)$. The choice for the mass spectrum peak indexing is the same as the one commonly reported in literature [37-48]. $\mathrm{Mg}^{2+}$ cations are associated with the peaks at $12,12.5$ and $13 \mathrm{Da}$, due to its three isotopes (fig. 5(b)). However, only the principal isotope ${ }^{24} \mathrm{Mg}^{2+}$ (natural abundance: $79 \%$ ) can be clearly distinguished here at $12 \mathrm{Da}\left({ }^{24} \mathrm{Mg}^{2+}\right.$ signal-to-noise ratio $\left.\approx 4\right) .{ }^{25} \mathrm{Mg}^{2+}$ and ${ }^{26} \mathrm{Mg}^{2+}$ are partially buried inside the background noise, indicating that the $\mathrm{Mg}$ concentration is close to the APT detection limit $\left(\sim 10^{19} \mathrm{~cm}^{-3}\right)$ [49]. $\mathrm{Ge}^{2+}$ cations naturally occur in five isotopes forming peaks at $35,36,36.5,37$ and $38 \mathrm{Da}$ in the mass spectrum (fig. 5(c)). However, the presence of the two thermal tails associated to $\mathrm{Ga}^{2+}$ cations appearing at 34.5 and 35.5 Da leads to an overestimation of the number of Ge atoms detected. To overcome all these limitations, a local background noise correction was performed in order to calculate the number of $\mathrm{Mg}$ and $\mathrm{Ge}$ atoms. The background signal was measured before and after the $\mathrm{Mg}^{2+}$ and $\mathrm{Ge}^{2+}$ peaks, and the average background noise level was subtracted to the peaks before quantification of the atomic concentration.

Despite APT providing a near-atomic scale spatial resolution, this technique still has some limitations to provide an accurate and reliable quantification of concentrations. Deviations from the stoichiometric composition were recently reported in the case of binary (i.e. GaN), ternary (i.e. AIGaN, InGaN) and heavily doped III-N semiconductors (i.e. BGaN) [38-45]. The origin of such biases is the result of the complex phenomena occurring at the tip surface during laser assisted field evaporation processes (i.e. short-range diffusion phenomena, DC-field induced preferential evaporation of metallic elements, molecular dissociation reactions, etc.). In order to mitigate such issue, the biased concentration profiles supplied by APT were corrected a posteriori using the information provided by SIMS. Briefly, it was assumed that SIMS provides an accurate quantification of the number of atoms present in each single layer of the LED stack, as indicated in fig. 4. Then, such quantities were used to normalize the APT concentration profiles, without changing the spatial distribution of atoms provided by this technique. The main limitation of this approach is that it cannot be applied for doping concentrations below the APT quantification limit $\left(\sim 10^{19} \mathrm{~cm}^{-3}\right.$ for the CAMECA FlexTAP system used in this study). Results are reported in fig. 5(d), where the dopant concentration profiles obtained in the TJ are represented. Data reveal a $\sim 20 \mathrm{~nm}$ thick heavy Ge-doped region $\left(\sim 1 \times 10^{20} \mathrm{~cm}^{-3}\right)$, corresponding to the $\mathrm{n}^{++} \mathrm{GaN}$ layer. The $\mathrm{Mg}$ concentration peak corresponding to 
the $10 \mathrm{~nm}$ thick $\mathrm{p}^{++}$layer appears overlapped with the Ge signal at the TJ, which confirms the segregation of $\mathrm{Mg}$ into the $\mathrm{n}^{++}$layer. It must be reminded that the diffusion of $\mathrm{Mg}$ and $\mathrm{Ge}$ in $\mathrm{GaN}$ during the growth process are expected to be very different. Mg is known to segregate strongly during the growth of GaN [5051]. On the contrary, Ge is incorporated in layer and it does not perturb the surface growth kinetics [16]. Note that, after incorporation in the lattice, neither Mg nor Ge are expected to diffuse unless the layers are annealed at very high temperature $\left(>900^{\circ} \mathrm{C}\right)[52]$. The maximum dopant concentrations reached at the TJ are $5 \times 10^{20} \mathrm{~cm}^{-3}$ for $\mathrm{Mg}$ and $2 \times 10^{20} \mathrm{~cm}^{3}$ for Ge. These peak values are higher than those provided by the SIMS profiles because of the smoothing induced by the low spatial resolution of SIMS (see fig. 4). In opposition to previous observation on heavy Mg-doped GaN, no Mg clustering is observed here [48]. In fact, Mg clustering in MOCVD grown layers appears associated with the generation of pyramidal inversion domains [53], which occur when the $\mathrm{Mg}$ concentration is in the mid $10^{19} \mathrm{~cm}^{-3}$ range [54]. SIMS results show that the $\mathrm{Mg}$ concentration of the $\mathrm{p}$-type layer was in the range of $1-3 \times 10^{19} \mathrm{~cm}^{-3}$, so that it is reasonable to assume that pyramidal inversion domains were not formed during the growth. Furthermore, the heavily $\mathrm{Mg}$ doped layer at the $\mathrm{TJ}\left([\mathrm{Mg}]=1 \times 10^{20} \mathrm{~cm}^{-3}\right)$ is only $10 \mathrm{~nm}$ thick, so that the probability of observing clustering is relatively small.

(a)

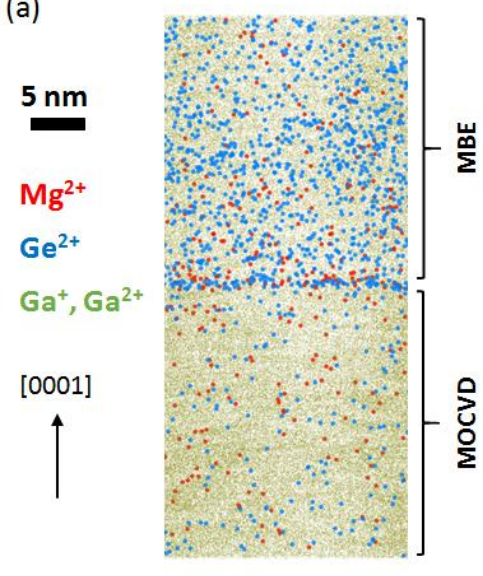

(b)

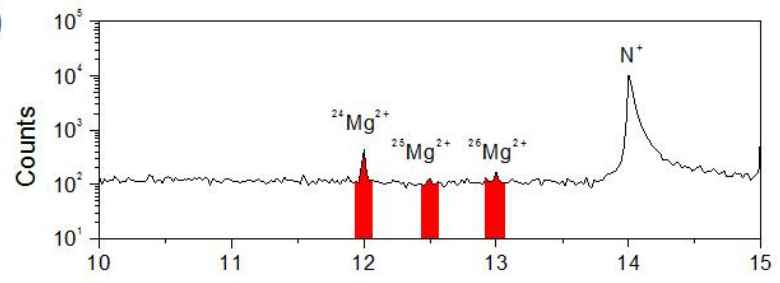

(c)

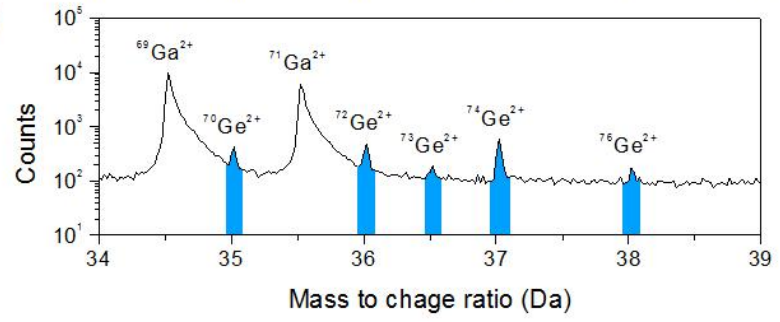

(d)

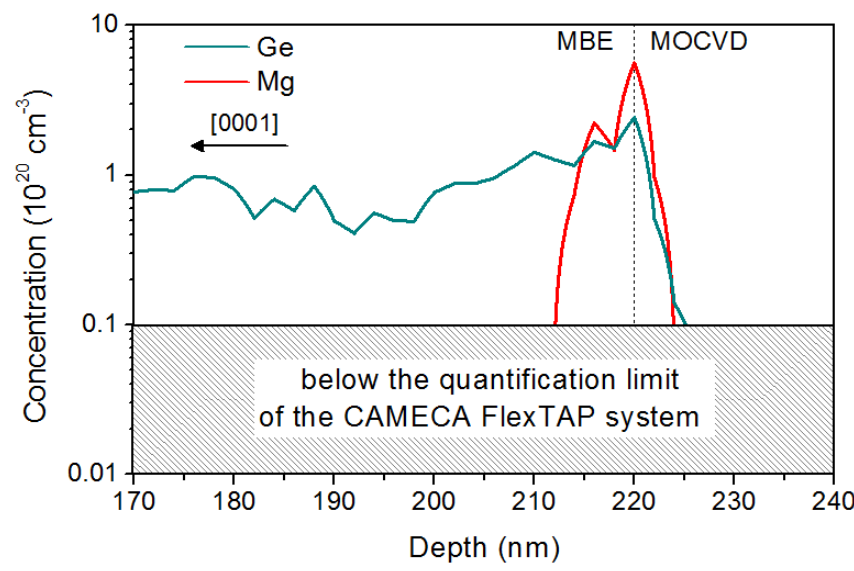


Fig. 5: (a) Atom probe 3D reconstruction of the tunnel junction. $\mathrm{Mg}^{2+}$ ions are shown in red, $\mathrm{Ge}^{2+}$ ions appear in blue, while $\mathrm{Ga}^{+}$and $\mathrm{Ga}^{2+}$ ions are represented in green. The image corresponds to a $20 \times 30 \times 50 \mathrm{~nm}^{3}$ volume. $(b, c)$ Detail of the atom probe mass spectrum showing the peaks associated to $\mathrm{Mg}$ and $\mathrm{Ge}$. (d) $\mathrm{Mg}$ and Ge concentration profiles obtained combining the spatial distribution of dopants provided by APT with the SIMS concentration profiles.

\section{(e) Electron Holography}

APT and SIMS provide chemical information but cannot determine the electrical activity of the dopants. To be able to visualize the relative activity, off-axis electron holography was performed. Here, an electron biprism was used to create an interference pattern known as the hologram. A coherent electron wave that passes through the specimen was interfered with another that passes through vacuum. From a Fourier reconstruction procedure, information about the phase change of these electrons can be retrieved. This is sensitive to the local electrostatic potential due to the presence of active dopants $V_{\text {dopant }}$ and the mean inner potential (MIP) $V_{M I P}$. The phase change $\Delta \varphi(x, y)$ of an electron that passes through a specimen is given by the following equation [55]:

$$
\Delta \varphi(x, y)=C_{E} \int_{0}^{t} V_{E}(x, y, z) d z, \quad \text { (eq. 2) }
$$

where: $V_{E}=V_{M I P}+V_{\text {dopant }} ; C_{E}$ is a constant dependent on the operating voltage of the TEM, which equals to $7.3 \mathrm{mrad} \cdot \mathrm{nm}^{-1} \cdot \mathrm{V}^{-1}$ for $200 \mathrm{kV}$ electrons; $t$ is the thickness of the specimen. The value of the $V_{M I P}$ for GaN was calculated by density functional theory (DFT) and is found to be $16.8 \mathrm{~V}$ [56].

To reduce the presence of the "inactive" thickness, a relatively thick (300 nm) specimen was prepared. To avoid diffraction contrast the specimen was tilted "edge on" a few degrees from the [0120] zone axis such that the information about the different doped layers was not lost in projection. To improve the signal-tonoise ratio in the reconstructed phase images whilst reducing the effects of specimen damage and charging [48], stacks of electron holograms were acquired (32 electron holograms each acquired for 8 seconds) using a low beam current. Each electron hologram used a fringe spacing of $1.2 \mathrm{~nm}$, which was reconstructed using the Holoview software to provide a nominal spatial resolution of $2.4 \mathrm{~nm}$ [29].

The reconstructed phase is shown in fig. 6(a). The phase image is sensitive to both the MIP and the active dopants. As the $\ln _{y} \mathrm{Ga}_{1-\mathrm{y}} \mathrm{N}$ and $\mathrm{GaN}$ regions have very similar values of MIP [57], in the absence of diffraction contrast the phase contrast can be directly interpreted in terms of the conduction band of the stack. The 
$\mathrm{Al}_{\mathrm{y}} \mathrm{Ga}_{1-\mathrm{y}} \mathrm{N}$ EBL appears darker as this has a lower value of MIP. In fig. 6(a) the piezoelectric fields in the five $\mathrm{In}_{\mathrm{y}} \mathrm{Ga}_{1-\mathrm{y}} \mathrm{N}$ QWs can be observed; however, this experiment was not designed to resolve these structures as a thinner specimen would be required to reduce the diffraction contrast at the $G a N / \ln _{y} G a_{1-y} N$ interfaces [58]. In the position of the TJ, a clear phase contrast change that marks the transition between the Mg- and Ge-doped GaN layers can be observed. In these experiments quantification of the phase has not been attempted as it is known that the measured potential is much less than expected for doped GaN specimens [59]. Additional attempts to quantify the potentials in p-n junction specimens has proved difficult [60]. However, in these thick specimens the measurement of the depletion width is accurate [61]. Finally, from the phase image a $\Delta \varphi$ profile has been extracted across the TJ and shown in fig. $6(\mathrm{~b})$, where the depletion width $w_{d}$ can be directly measured. Assuming that the limitations from the spatial resolution provides a maximum value of the depletion width, the experimental data reveals a value of depletion width $w_{d} \approx 7 \mathrm{~nm}$, estimated from $10 \%$ to $90 \%$ thresholds.

(a)

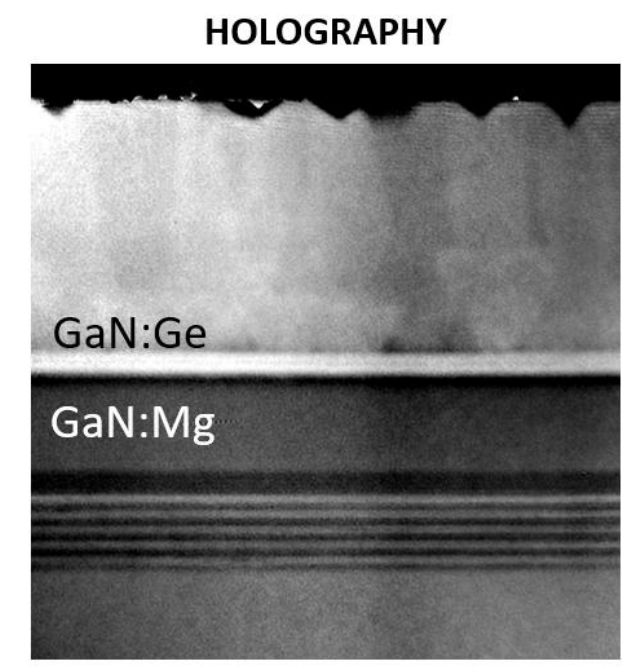

(b)

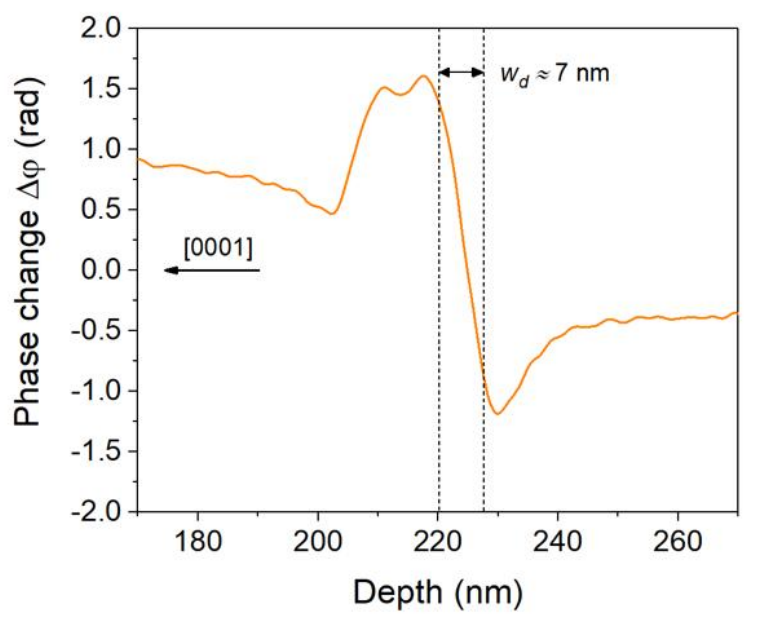

Fig. 6: (a) Phase-contrast image from dark-field electron holography. The interface between MOCVD grown GaN and MBE grown GaN is here well resolved. (b) Profile of the phase change $\Delta \varphi$ across the TJ. The depletion width $w_{d}$ estimated from $10 \%$ to $90 \%$ thresholds is equal to $\sim \mathrm{nm}$.

\section{(f) Band Diagram Simulations}

In order to study the effect of the dopant distribution on the electrical performance of the junction, we have compared the band diagram of the nominal stack (calculation presented in fig. 1(b)) with the band diagram taking into account the doping profiles measured in fig. 5(d). This second calculation considered the complete structure and was performed in the same manner as described for fig. 1(b), but not for the 
dopant distribution, where the nominal values were replaced by the experimentally extracted profile of dopants. A view of both band diagrams around the $T J$ is shown in fig. 7. From the simulations the width of the depletion region $w_{d}$ can be measured to be $\sim 7 \mathrm{~nm}$. This result is in good agreement with the electron holography measurement (see fig. 6). The most apparent effect of $\mathrm{Mg}$ segregation at the MOCVD/MBE interface is to move the depletion layer by $\sim 1 \mathrm{~nm}$ toward the sample surface. Note that the depletion region width in the real structure is very close to the expected width in the nominal design despite the very different distribution of dopants.

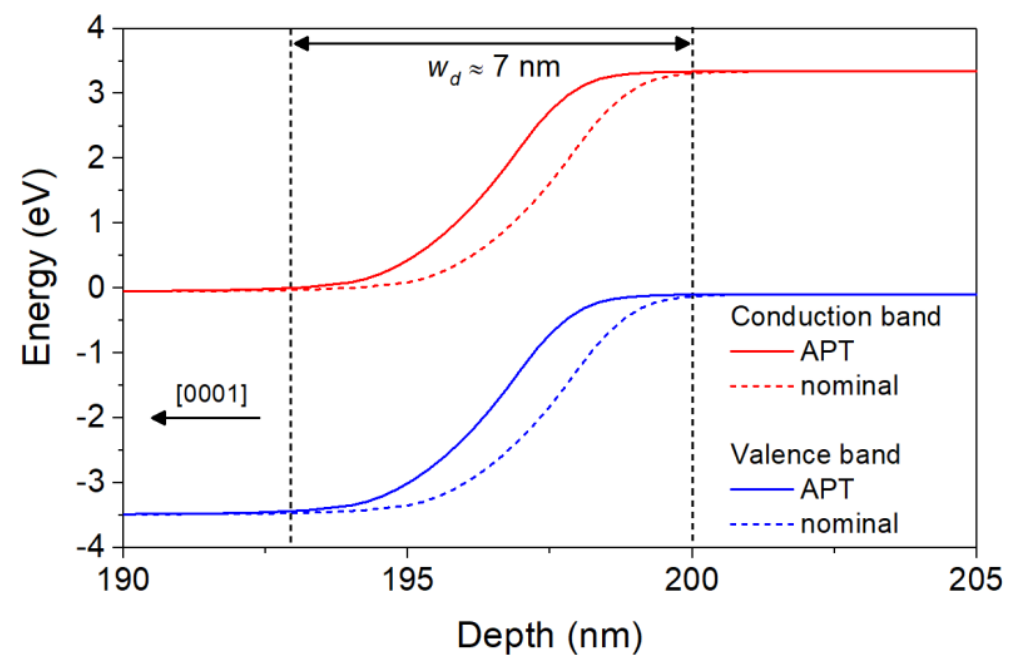

Fig. 7: Detail of the band diagram around the r-point in correspondence of the MOCVD/MBE interface. Both the nominal LED structure and a second one derived by APT observations were analyzed. The junction temperature is $300 \mathrm{~K}$ and no bias is applied.

\section{Conclusions}

In conclusion, we have presented a multi-microscopy study of a TJ grown on top of an $\ln _{\mathrm{y}} \mathrm{Ga}_{1-\mathrm{y}} \mathrm{N} / \mathrm{GaN}$ LED. The TJ investigated here consists of Ge-doped layers regrown by ammonia-MBE over Mg-doped layers grown by MOCVD. A quantitative atomic-scale picture of Mg- and Ge-doping at the $\mathrm{TJ}$ is obtained. The high mobility of $\mathrm{Mg}$ atoms results in their segregation and diffusion into the $\mathrm{n}$-doped GaN. The active dopant distribution revealed by electron holography shows a depletion region $\sim 7 \mathrm{~nm}$ wide across the TJ. Calculations based on the experimental doping profiles provided by atom probe tomography and secondary ion mass spectrometry are in agreement with the electron holography observations. $\mathrm{Mg}$ diffusion phenomena result in a $\sim 1 \mathrm{~nm}$ shift of the depletion region towards the $\mathrm{n}$-doped $\mathrm{GaN}$, although such dopant distribution appears very different compared to the nominal one. These results not only demonstrate the capabilities of the different techniques in revealing the atomic-scale dopant distribution, but also represent 
a significant gain in understanding the diffusion phenomena associated to the growth of hybrid $\mathrm{Mg} / \mathrm{Ge}$ doped tunnel junctions.

\section{Acknowledgments}

This work has been funded through projects DUVET (ANR project), GaNeX (GaNeX belongs to the public funded "Investissement d'Avenir" program managed by the French ANR agency) and INMoSt (ANR-19CE08-0025). The experiments have been performed on the PFNC Nanocharactersiation centre at Minatec, Grenoble.

\section{Bibliography}

[1] E. F. Schubert, J. K. Kim, Solid-state light sources getting smart, Science 308(5726), 1274-1278 (2005).

[2] E. R. Glaser, W. E. Carlos, G. C. B. Braga, J. A. Freitas Jr., W. J. Moore, B. V. Shanabrook, R. L. Henry, A. E. Wickenden, D. D. Koleske, H. Obloh, P. Kozodoy, S. P. DenBaars, U. K. Mishra, Magnetic resonance studies of Mg-doped GaN epitaxial layers grown by organometallic chemical vapor deposition, Phys. Rev. B 65, 085312 (2002).

[3] S. R. Jeon, Y. H. Song, H. J. Jang, G. M. Yang, S. W. Hwang, S. J. Son, Lateral current spreading in GaNbased light-emitting diodes utilizing tunnel contact junctions, Appl. Phys. Lett. 78(21), 3265-3267 (2001).

[4] M. Malinverni, D. Martin, N. Grandjean, InGaN based micro light emitting diodes featuring a buried GaN tunnel junction, Appl. Phys. Lett. 107(5), 051107 (2015).

[5] A. G. Sarwar, B. J. May, J. I. Deitz, T. J. Grassman, D. W. McComb, R. C. Myers, Tunnel junction enhanced nanowire ultraviolet light emitting diodes, Appl. Phys. Lett. 107(10), 101103 (2015).

[6] Y. Zhang, S. Krishnamoorthy, F. Akyol, S. Bajaj, A. A. Allerman, M. W. Moseley, A. M. Armstrong, S. Rajan, Tunnel-injected sub-260 nm ultraviolet light emitting diodes, Appl. Phys. Lett. 110(20), 201102 (2017).

[7] E. C. Young, B. P. Yonkee, F. Wu, S. H. Oh, S. P. DenBaars, S. Nakamura, J. S Speck, Hybrid tunnel junction contacts to III-nitride light-emitting diodes, Appl. Phys. Expr. 9(2), 022102 (2016).

[8] J. T. Leonard, E. C. Young, B. P. Yonkee, D. A. Cohen, T. Margalith, S. P. DenBaars, J. S Speck, S. Nakamura, Demonstration of a III-nitride vertical-cavity surface-emitting laser with a III-nitride tunnel junction intracavity contact, Appl. Phys. Lett. 107(9), 091105 (2015).

[9] H. Y. Ryu, I. G. Choi, H. S. Choi, J. I. Shim, Investigation of light extraction efficiency in AlGaN deepultraviolet light-emitting diodes, Appl. Phys. Expr. 6(6), 062101 (2013).

[10] F. Akyol, S. Krishnamoorthy, S. Rajan, Tunneling-based carrier regeneration in cascaded GaN light emitting diodes to overcome efficiency droop, Appl. Phys. Lett. 103(8), 081107 (2013).

[11] W. Götz, R. S. Kern, C. H. Chen, H. Liu, D. A. Steigerwald, R. M. Fletcher, Hall-effect characterization of III-V nitride semiconductors for high efficiency light emitting diodes, Mater. Sci. Eng. B 59(1-3), 211-217. (1999).

[12] S. Fritze, A. Dadgar, H. Witte, M. Bügler, A. Rohrbeck, J. Bläsing, H. Hoffmann, A. Krost, High Si and Ge n-type doping of GaN doping-Limits and impact on stress, Appl. Phys. Lett. 100(12), 122104 (2012).

[13] C. Nenstiel, M. Bügler, G. Callsen, F. Nippert, T. Kure, S. Fritze, A. Dadgar, H. Witte, J. Bläsing, A. Krost, A. Hoffmann, Germanium-the superior dopant in n-type GaN, Phys. Stat. Sol. - Rapid Research Letters 9(12), 716-721 (2015). 
[14] A. Ajay, J. Schörmann, M. Jiménez-Rodriguez, C. B. Lim, F. Walther, M. Rohnke, I. Mouton, L. Amichi, C. Bougerol, M. I. Den Hertog, M. Eickhoff, E. Monroy, Ge doping of GaN beyond the Mott transition, Jour. Phys. D: Appl. Phys. 49(44), 445301 (2016).

[15] R. Blasco, A. Ajay, E. Robin, C. Bougerol, K. Lorenz, L. C. Alves, I. Mouton, L. Amichi, A. Grenier, E. Monroy, Electrical and optical properties of heavily Ge-doped AlGaN, J. Phys. D: Appl. Phys. 52, 125101 (2019).

[16] H. Okumura, D. Martin, M. Malinverni, N. Grandjean, Backward diodes using heavily Mg-doped GaN growth by ammonia molecular-beam epitaxy, Appl. Phys. Lett. 108(7), 072102 (2016).

[17] S. Neugebauer, M. P. Hoffmann, H. Witte, J. Bläsing, A. Dadgar, A. Strittmatter, T. Niermann, M. Narodovitch, M. Lehmann, All metalorganic chemical vapor phase epitaxy of $p / n-G a N$ tunnel junction for blue light emitting diode applications, Appl. Phys. Lett. 110(10), 102104 (2017).

[18] X. Yan, W. Li, S. M. Islam, K. Pourang, H. Xing, P. Fay, D. Jena, Polarization-induced Zener tunnel diodes in GaN/InGaN/GaN heterojunctions, Appl. Phys. Lett. 107(16), 163504 (2015).

[19] H. Morkoc, Nitride Semiconductor Devices, chap. 1, Wiley-VCH (2012).

[20] W. Li, K. Nomoto, K. Lee, S. M. Islam, Z. Hu, M. Zhu, X. Gao, J. Xie, M. Pilla, D. Jena, H. G. Xing, Activation of buried p-GaN in MOCVD-regrown vertical structures, Appl. Phys. Lett. 113(6), 062105 (2018).

[21] C. G. Van de Walle, C. Stampfl, J. Neugebauer, Theory of doping and defects in III-V nitrides, J. Cryst. Growth 189, 505-510. (1998).

[22] V. Fan Arcara, B. Damilano, G. Feuillet, A. Courville, S. Chenot, J. Y. Duboz, (Ga, In)N/GaN light emitting diodes with a tunnel junction and a rough n-contact layer grown by metalorganic chemical vapor deposition, AIP Advances 9.5, 055101 (2019).

[23] V. Fan Arcara, B. Damilano, G. Feuillet, S. Vézian, K. Ayadi, S. Chenot, J. Y.Duboz, Ge doped GaN and $A I_{0.5} \mathrm{Ga}_{0.5} \mathrm{~N}$-based tunnel junctions on top of visible and UV light emitting diodes, Jour. Appl. Phys. 126(22), 224503 (2019).

[24] S. Birner, T. Zibold, T. Andlauer, T. Kubis, M. Sabathil, A. Trellakis, and P. Vogl, nextnano: General Purpose 3-D Simulations, IEEE Transactions on Electron Devices 54, 2137-42 (2007).

[25] T. Suski, G. Staszczak, K. P. Korona, P. Lefebvre, E. Monroy, P. A. Drozdz, G. Muzioł, C. Skierbiszewski, M. Kulczykowski, M. Matuszewski, E. Grzanka, S. Grzanka, K. Pieniak, K. Gibasiewicz, A. Khachapuridze, J. Smalc-Koziorowska, L. Marona, P. Perlin, Switching of exciton character in double InGaN/GaN quantum wells, Physical Review B, 98(16), 165302 (2018).

[26] S. Padalkar , J.R. Riley , Q. Li , G.T. Wang, L.J. Lauhon , Lift-out procedures for atom probe tomography targeting nanoscale features in core-shell nanowire heterostructures, Phys. Status Solidi C 11 (3-4), 656 (2014).

[27] I. Blum , F. Cuvilly , W. Lefebvre-Ulrikson, Chapter four-Atom probe sample preparation, in: Atom Probe Tomography, Academic Press, 97-121 (2016).

[28] J. Bogdanowicz, A. Kumar, C. Fleischmann, M. Gilbert, J. Houard, A. Vella, W. Vandervorst, Laserassisted atom probe tomography of semiconductors: The impact of the focused-ion beam specimen preparation, Ultramicroscopy 188, 19 (2018).

[29] V.Boureau, R. McLeod, B. Mayall, D. Cooper, Off-axis electron holography combining summation of hologram series with double-exposure phase-shifting: theory and application, Ultramicroscopy, 193, 52-63 (2018).

[30] www.probion.fr.

[31] D. Larson, B. Gault, B. Geiser, F. De Geuser, F. Vurpillot, Atom probe tomography spatial reconstruction: Status and directions, Current Opinion in Solid State and Materials Science 17, 236-247 (2013). 
[32] S. Pezzagna, P. Vennéguès, N. Grandjean, J. Massies, Polarity inversion of GaN (0001) by a high Mg doping, Journal of Crystal Growth 269(2-4), 249-256. (2004).

[33] K. S. Son, D. G. Kim, H. K. Cho, K. Lee, S. Kim, K. Park, Formation of V-shaped pits in GaN thin films grown on high temperature GaN, J. Cryst. Growth, 261(1), 50-54. (2004).

[34] K. S. Son, D. G. Kim, H. K. Cho, K. Lee, S. Kim, K. Park, J. Kim, Effect of growth temperature and Si doping on the microstructure of GaN thin films grown on high temperature GaN, Phys. Stat. Sol. (c) 7, 2095-2098. (2003).

[35] S. Fritze, A. Dadgar, H. Witte, M. Bügler, A. Rohrbeck, J. Bläsing, A. Hoffmann, A. Krost, High Si and Ge n-type doping of GaN doping-Limits and impact on stress, Appl. Phys. Lett. 100(12), 122104 (2012).

[36] M. N. Fireman, G. L'Heureux, F. Wu, T. Mates, E. C. Young, J. S. Speck, High germanium doping of GaN films by ammonia molecular beam epitaxy. J. Cryst. Growth 508, 19-23 (2019).

[37] Y. Oshima, Properties of Ge-doped, high-quality bulk GaN crystals fabricated by hydride vapor phase epitaxy, Journal of Crystal Growth 312, 3569-3573 (2010).

[38] E. Di Russo, I. Blum, J. Houard, M. Gilbert, G. Da Costa, D. Blavette, L. Rigutti, Compositional accuracy of atom probe tomography measurements in GaN: Impact of experimental parameters and multiple evaporation events, Ultramicroscopy 187, 126 (2018).

[39] L. Mancini, N. Amirifar, D. Shinde, I. Blum, M. Gilbert, A. Vella, F. Vurpillot, W. Lefebvre, R. Lardé, E. Talbot, P. Pareige, X. Portier, A. Ziani, C. Davesnne, C. Durand, J. Eymery, R. Butté, J.-F. Carlin, N. Grandjean, L. Rigutti, Composition of wide bandgap semiconductor materials and nanostructures measured by atom probe tomography and its dependence on the surface electric field, J. Phys. Chem. C, 118, 24136 (2014).

[40] E. Di Russo, N. Cherkashin, M. Korytov, A. E. Nikolaev, A. V. Sakharov, A. F. Tsatsulnikov, B. Bonef, I. Blum, J. Houard, G. Da Costa, D. Blavette, L. Rigutti, Compositional accuracy in atom probe tomography analyses performed on III-N light emitting diodes, J. Appl. Phys. 126.12, 124307 (2019).

[41] E. Di Russo, F. Moyon, N. Gogneau, L. Largeau, E. Giraud, J.-F. Carlin, N. Grandjean, J. M. Chauveau, M. Hugues, I. Blum, W. Lefebvre, F. Vurpillot, D. Blavette, L. Rigutti, Composition metrology of ternary semiconductor alloys analyzed by Atom Probe Tomography, J. Phys. Chem. C 122.29, 16704-16714 (2018).

[42] L. Rigutti, B. Bonef, J. Speck, F. Tang, R. A. Oliver, Atom probe tomography of nitride semiconductors, Scripta Materialia 148, 75-81 (2018).

[43] B. Bonef, R. Cramer, J. S. Speck, Nanometer scale composition study of MBE grown BGaN performed by atom probe tomography, J. Appl. Phys. 121(22), 225701 (2017).

[44] E. Di Russo, Study of the physical mechanisms leading to compositional biases in atom probe tomography of semiconductors, Ph.D thesis, Université de Rouen (2018).

[45] R. J. H. Morris, R. Cuduvally, D. Melkonyan, C. Fleischmann, M. Zhao, L. Arnoldi, P. van der Heide, and W. Vandervorst, Toward accurate composition analysis of GaN and AlGaN using atom probe tomography, J. Vac. Sci. Technol. B 36(3), 03F130 (2018).

[46] R. J. H. Morris, R. Cuduvally, D. Melkonyan, M. Zhao, P. van der Heide, W. Vandervorst, Atom probe of GaN/AIGaN heterostructures: The role of electric field, sample crystallography and laser excitation on quantification, Ultramicroscopy 206, 112813 (2019).

[47] Y. Shimizu, H. Takamizawa, Y. Kawamura, M. Uematsu, T. Toyama, K. Inoue, E. E. Haller, K. M. Itoh, Y. Nagai, Atomic-scale characterization of germanium isotopic multilayers by atom probe tomography, J. Appl. Phys. 113, 026101 (2013).

[48] L. Amichi, I. Mouton, E. Di Russo, V. Boureau, P. Vennegues, P. De Mierry, A. Grenier, P.-H. Jouneau, C. Bougerol, D. Cooper, Correlative investigation of $\mathrm{Mg}$ doping in GaN layers grown at different temperatures by atom probe tomography and off-axis electron holography, Nanotechnology 31(4), 045702 (2019). 
[49] A. M. Siladie, L. Amichi, N. Mollard, I. Mouton, B. Bonef, C. Bougerol, A. Grenier, E. Robin, P.-H. Jouneau, N. Garro, A. Cros, B. Daudin, Dopant radial inhomogeneity in Mg-doped GaN nanowires, Nanotechnology 29(25), 255706 (2018).

[50] K. Köhler, R. Gutt, J. Wiegert, L. Kirste, Diffusion of Mg dopant in metal-organic vapor-phase epitaxy grown $G a N$ and $A l_{x} G a_{1-x} N$, J. Appl. Phys. 113(7), 073514 (2013).

[51] E. Monroy, T. Andreev, P. Holliger, E. Bellet-Amalric, T. Shibata, M. Tanaka, B. Daudin, Modification of GaN (0001) growth kinetics by Mg doping, Appl. Phys. Lett. 84(14), 2554-2556 (2004).

[52] R. G. Wilson, S. J. Pearton, C. R. Abernathy, J. M. Zavada, Thermal stability of implanted dopants in GaN, Appl. Phys. Lett. 66(17), 2238-2240 (1995).

[53] S. E. Bennett, R. M. Ulfig, P. H. Clifton, M. J. Kappers, J. S. Barnard, C. J. Humphreys, R. A. Oliver, Atom probe tomography and transmission electron microscopy of a Mg-doped AlGaN/GaN superlattice, Ultramicroscopy, 111(3), 207-211 (2011).

[54] M. Leroux, P. Vennegues, S. Dalmasso, M. Benaissa, E. Feltin, P. De Mierry, B. Beaumont, B. Damilano, N. Grandjean, P. Gibart, Structural Defects and Relation with Optoelectronic Properties in Highly Mg-Doped GaN, Phys. Stat. Sol. (a) 192(2), 394-400 (2002).

[55] M. McCartney, D. Smith, R. Hull, J. Bean, E. Voelkl, B. Frost, Direct observation of potential distribution across Si/Si p-n junctions using off-axis electron holography, Appl. Phys. Lett. 65(20), 2603-2605 (1994).

[56] P. Kruse, M. Schowalter, D. Lamoen, A. Rosenauer, D. Gerthsen, Determination of the mean inner potential in III-V semiconductors, Si and Ge by density functional theory and electron holography, Ultramicroscopy 106, 105-13 (2006).

[57] K. Song, C. T. Koch, J. K. Lee, D. Y. Kim, , J. K. Kim, A. Parvizi, W. Y. Jung, C. G. Park, H. J. Jeong, H. S. Kim, Y. Cao, T. Yang, L.-Q. Chen, S. H. Oh, Correlative high-resolution mapping of strain and charge density in a strained piezoelectric multilayer, Advanced Materials Interfaces, 2(1), 1400281 (2015).

[58] B. Haas, J. L. Rouviere, V. Boureau, R. Berthier, D. Cooper, Direct comparison of off-axis holography and differential phase contrast for the mapping of electric fields in semiconductors by transmission electron microscopy, Ultramicroscopy, 198, 58-72 (2019).

[59] J. B. Park, T. Niermann, D. Berger, A. Knauer, I. Koslow, M. Weyers, M. Kneissl, M. Lehmann, Impact of electron irradiation on electron holographic potentiometry, Appl. Phys. Lett. 105(9), 094102 (2014).

[60] L. Amichi, Study of p-type doping in GaN nanostructures by correlation between atom probe tomography and off-axis electronic holography, PhD Thesis, Université de Grenoble (2018).

[61] P. K. Somodi, A. C. Twitchett-Harrison, P. A. Midgley, B. E. Kardynat, C. H. W. Barnes, R. E. DuninBorkowski, Finite element simulations of electrostatic dopant potentials in thin semiconductor specimens for electron holography. Ultramicroscopy 134, 160-166 (2013). 